\title{
Coding observational data: A software solution
}

\author{
OTTO H. MACLIN and M. KIMBERLY MACLIN \\ University of Northern Iowa, Cedar Falls, Iowa
}

\begin{abstract}
The analysis of observational data, particularly from social interactions, is often made difficult by the process of transcribing the verbal and behavioral interactions, having observers code on the transcriptions, and then transferring those codes to a statistical analysis program. The advent of computeraided coding schemes has, to some extent, eliminated this laborious and time-consuming process. However, existing programs may not fulfill the needs of all of the researchers interested in this methodology, and furthermore, the expense of current systems may put these tools out of reach for some. The Observational Data Coding System (ODCS) software package presented here is a flexible coding tool designed to make efficient the process of coding observational data. A control panel allows the researcher to customize the program and coding system to suit his or her research questions. A variety of media (including video, audio, image, and text) can be read directly into the Windows-based program; trained observers can then code a variety of factors via buttons on the screen while the file is playing. An example of using the program to analyze conversation is provided. ODCS is a flexible, free tool for the coding of observational data.
\end{abstract}

Many researchers in social psychology, behavioral analysis, comparative psychology, clinical psychology, and other research areas are interested in the systematic observation of behavior. Social interactions, spoken interchanges, individual and group behaviors (animal or human), and nonverbal communication are just a few such domains in which the systematic coding of behaviors is often necessary. Typically, this coding process has been necessarily laborious, requiring that every codable factor (e.g., utterances or behaviors) be transcribed into a written form (Allen \& Guy, 1974; Potter \& Wetherell, 1995).

Distilling real-life events into analyzable form often results in large volumes of data. Take, for example, the coding of conversations. If one considers that it is estimated that $30 \mathrm{sec}$ of natural conversation equals approximately 4 pages of transcription (Lampert \& Ervin-Tripp, 1993), one can imagine that coding a transcription from a fairly simple 15 -min interaction would yield 450 pages of transcription. In addition, the resulting transcription often bears little resemblance to the real-life event that was coded, and these transcriptions are often very difficult to read (Drew, 1995; Edwards \& Lampert, 1993). Depending on the level of technology used, videotapes

Portions of this article were presented at the Society for Computers in Psychology, Minneapolis, November 2004. The authors thank Gerald P. Ginsburg and Richard Weiner for helpful suggestions regarding the coding program itself and its utility to various research areas. Thanks are also due two anonymous reviewers for their feedback. Correspondence concerning the software can be addressed to O. H. MacLin, and correspondence regarding the conversation coding example can be directed to M. K. MacLin, both at the Department of Psychology, University of Northern Iowa, Cedar Falls, IA 50614 (e-mail: otto.maclin@uni.edu or kim.maclin@uni.edu). may need to be time stamped - a process that requires playing the tapes through a machine that records the exact time onto each frame. Trained coders then view or listen to the interaction (on video- or audiotape) and code (according to a predefined system) each utterance, behavior, or other variable of interest directly onto the transcription, while also stopping, rewinding, pausing, and fast forwarding these tapes. These codes then are tallied (for frequency) or computed (for duration) by hand and inputted into some sort of statistical analysis program. Not only is this process time consuming and labor intensive, but also, by virtue of its many steps, may introduce error at several levels. Applications of computer technology to the field of behavior coding not only are useful, but also are a necessary addition to this methodology, to ensure its continued success and scientific rigor.

There are a multitude of codable behaviors available in any given context. The burden is on the researcher to deem what is important, given the research questions, and to train the observers to extract that information from the passing stream of action. Bakeman and Gottman (1997) have noted that borrowing someone else's coding scheme rarely is appropriate, because the developing of coding schemes is very much tied to theory: They must represent the specific problem under study. Given the importance of code-specific schemes, it is critical that a software package have flexibility and the capacity for user-defined control over coding parameters.

The present software package is designed to allow for the coding of observational data in such a way as to eliminate the transcription and time-stamping process, preserving the context within which that behavior is coded in a cost-effective way. Farrell (1991) has noted that technology can dramatically improve data collection and analysis, particularly with regard to observational data, 
but that many people do not take advantage of the technology because of complex programs or a lower skill level. This program is designed to be useful to researchers with varying levels of computer skill. It should be noted that this program is not designed to replace the theoretical rigor and detailed specifications required for coding human or animal behavior. These are still necessary components when any computer coding system is used.

\section{SYSTEM OVERVIEW}

The Observational Data Coding System (ODCS) software package is designed to allow for the coding of video, audio, image, or text media. This material may have been collected in the field or in the laboratory. Alternatively, it could be commercials taped off of television, film clips, or content off of the Internet (from Web pages to chat room sessions). If the files to be coded are already in compatible electronic format, one can proceed directly to the use of the ODCS program after copying the necessary files to the computer's hard drive. Otherwise, video- and audiotapes need first to be transferred to electronic format. For videotapes, this can be accomplished by using one of several video transfer systems available (e.g., Dazzle Digital Video Creator or any number of VHS/DVD recorder options). The Dazzle, for example, captures full-screen video with synchronized audio from a VCR, camcorder, or TV. It digitally compresses the video segment by 200 times and automatically stores the video segment to a personal computer in MPEG format. Many new computers have Microsoft Movie Maker video-editing software included. Movie Maker can also be downloaded free from Microsoft. ${ }^{1}$ Instructions on how to use Movie Maker are also included at the Microsoft site. ${ }^{2}$ Video capture and transfer systems are particularly useful if one has "old" data sitting around on videotapes that one wants to reanalyze or simply preserve in electronic format. This is advantageous, because the quality of the video picture on a videotape cassette will deteriorate over time. Also, once tapes have been converted to MPEG format, they can be burned into a CD or DVD to allow for transportability and backup of videotaped sessions.

ODCS is a PC Windows-based program using menus, buttons, and selection boxes, accessible via a mouse. An Internet-based tutorial is provided to familiarize the user with the program (http://www.uni.edu/ maclin/software/ odcs/odcs_instructions.html). A control panel allows the user to design his or her own coding scheme, particular to the research questions under study.

The control panel allows the user to design a coding program that will collect the frequency, timing, duration, and sequence of events, as well as Likert scale data and yes-no entries (using the extra categories panel). The coder also designates whether he or she will be using focal (continuous) sampling, where each occurrence of the behavior is recorded together with its time of occur- rence or the time it started and ended. In fact, all codes are time stamped so that the stream of action is preserved.

The program allows coders to view the session as often as needed or as proscribed by the researcher. Therefore, many event-recording passes can be made through the same media file. For example, it may be desirable for the observer to code only one speaker at a time, one type of event, or certain durations of the video or audio file. When he or she then goes back to code other speaker(s) (or events, behaviors, or segments), those codes are appended to the designated data file. During the data transformation process, these codes can then be sorted to represent the codes in real-time order, using a program such as MS Excel.

An Internet browser is also available to the user from a drop-down menu option. This allows the user to link to the Web site for ODCS for updates, technical support, and additional information (when the computer is connected to the Internet).

\section{System Requirements}

The minimum system requirements needed to operate the coding system are as follows: a PC, supporting a current Windows operating system, with $12 \mathrm{MB}$ of RAM, $48 \mathrm{~K}$ of free hard disk space, sound capability (sound card and speakers), and a VGA video card (if source files are video). The necessary video player comes with the program.

\section{Language}

ODCS 1.0 was developed in Visual Basic 6.0. It may not be $100 \%$ compatible with Microsoft.NET framework.

\section{User Interface}

Welcome screen. A welcome screen gives general information regarding ODCS, and with a click, the user proceeds directly to the program's control panel.

Control panel. The program starts with a control panel, where the researcher configures the specific coding scheme, the data files to be used, and other relevant information needed for the coder to proceed (see Figure 1).

General information labels. Five text boxes are provided for the researcher to designate information to be collected about the coder and/or the coding session. For example, the researcher might want such information as the coder's name, the current date, the session identification number, and the study identification number to be collected. The researcher types these labels into each text box. Prior to beginning the actual coding session, the coder will be prompted to enter this information, which will be recorded to the beginning of the data file for that session.

Data files. The researcher must also designate three file specification boxes. The first is the output file designation, where the researcher will provide the path and file name for the file where the coding data will be writ- 


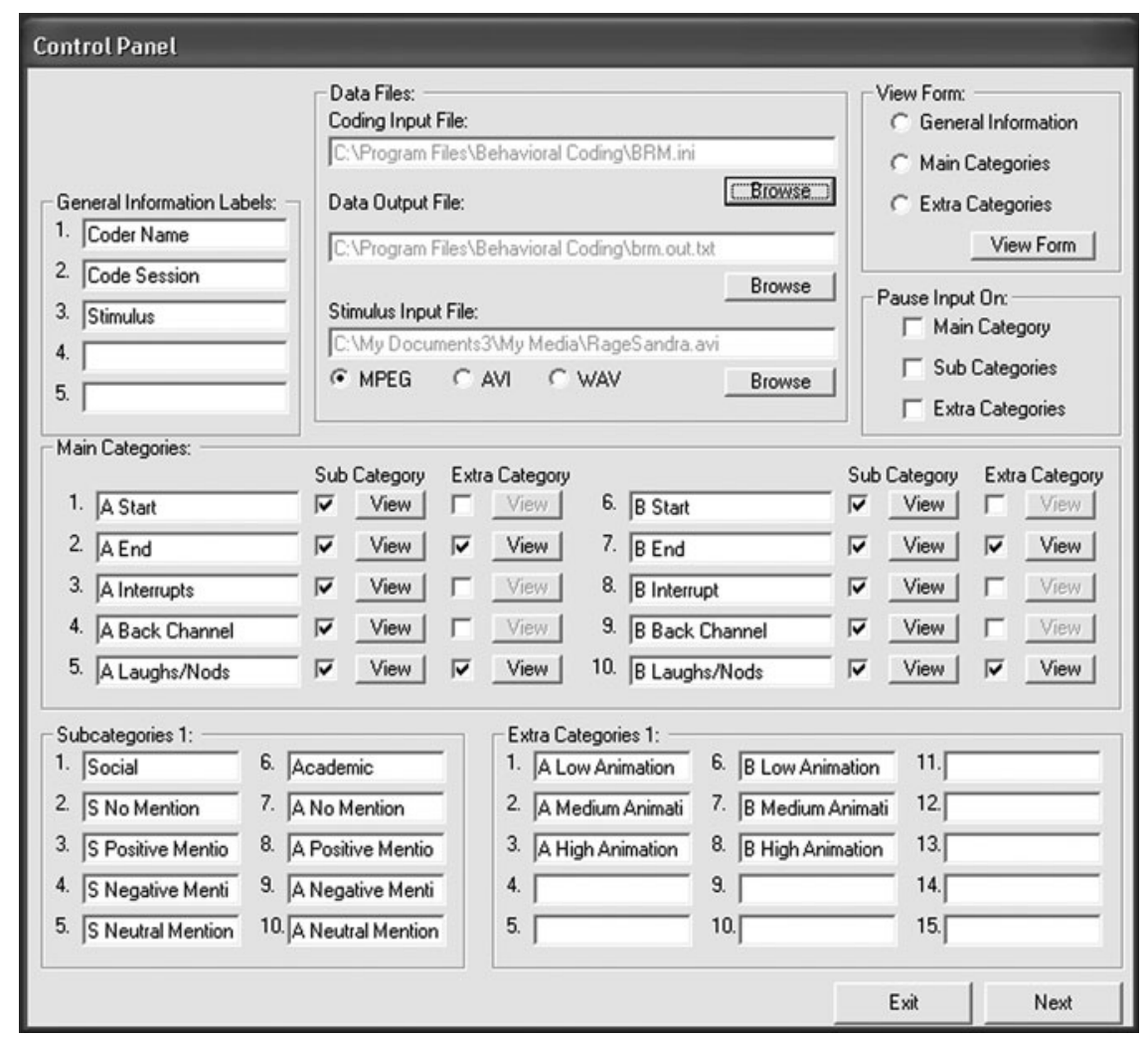

Figure 1. Control panel.

ten. The second is the stimulus input file, where the researcher will provide the path and file name for the observation session media file that is to be coded. The researcher indicates which type of media file (MPEG, AVI, TXT, or WAV) is to be used by clicking the appropriate radio button. Currently, these are the only files that are supported by ODCS. However, source code is available, and with some Visual Basic programming experience, it can be modified. In the third file specification box, the path and file name where the current control panel configurations will be saved are designated. An existing file with a previous control panel configuration can be selected as well.

Coding scheme. The following windows in the program allow the researcher to designate the particular coding scheme: main categories, subcategories, and extra categories. We have found that these parameters provide for a myriad of coding possibilities. Source code, though, is available for those who need to alter these basic characteristics.

Main categories. Ten main category coding options are available as text boxes. Each main category code can have linked to it 10 subcategory codes and 15 extra category codes. These categories are mutually exclusive.

Subcategories. Ten subcategory coding options are available. These subcategory codes are linked to specific main category codes, for a possible 100 subcategories
(10 main categories $\times 10$ subcategories). These categories are mutually exclusive.

Extra categories. Fifteen extra category coding options are available. These extra category codes are linked from specific main category codes, for a possible 150 extra categories (10 main categories $\times 15$ extra categories). Extra categories can be converted to Likert-type scales or yes/no responses. These can also be changed during the coding session to fit the needs of the coder.

Pause options. These three check boxes allow the researcher to designate whether the video or audio input is automatically paused when main category, subcategory, or extra category codes are used.

General information screen. Once the coding scheme has been specified, the researcher is prompted to save the settings. Next, the researcher proceeds to a screen where he or she is prompted to enter the information specified in general information labels (e.g., coder's name, etc.). Once completed, the user clicks next to go to the main coding screen, and this information is written to the data output file header, along with the current time and date.

Main coding screen. On the main coding screen, there are four cascading windows: (1) the video viewer, (2) the main categories panel, (3) the output viewer, and (4) the notes panel (see Figure 2). These windows can be repositioned anywhere on the screen, minimized, or re- 


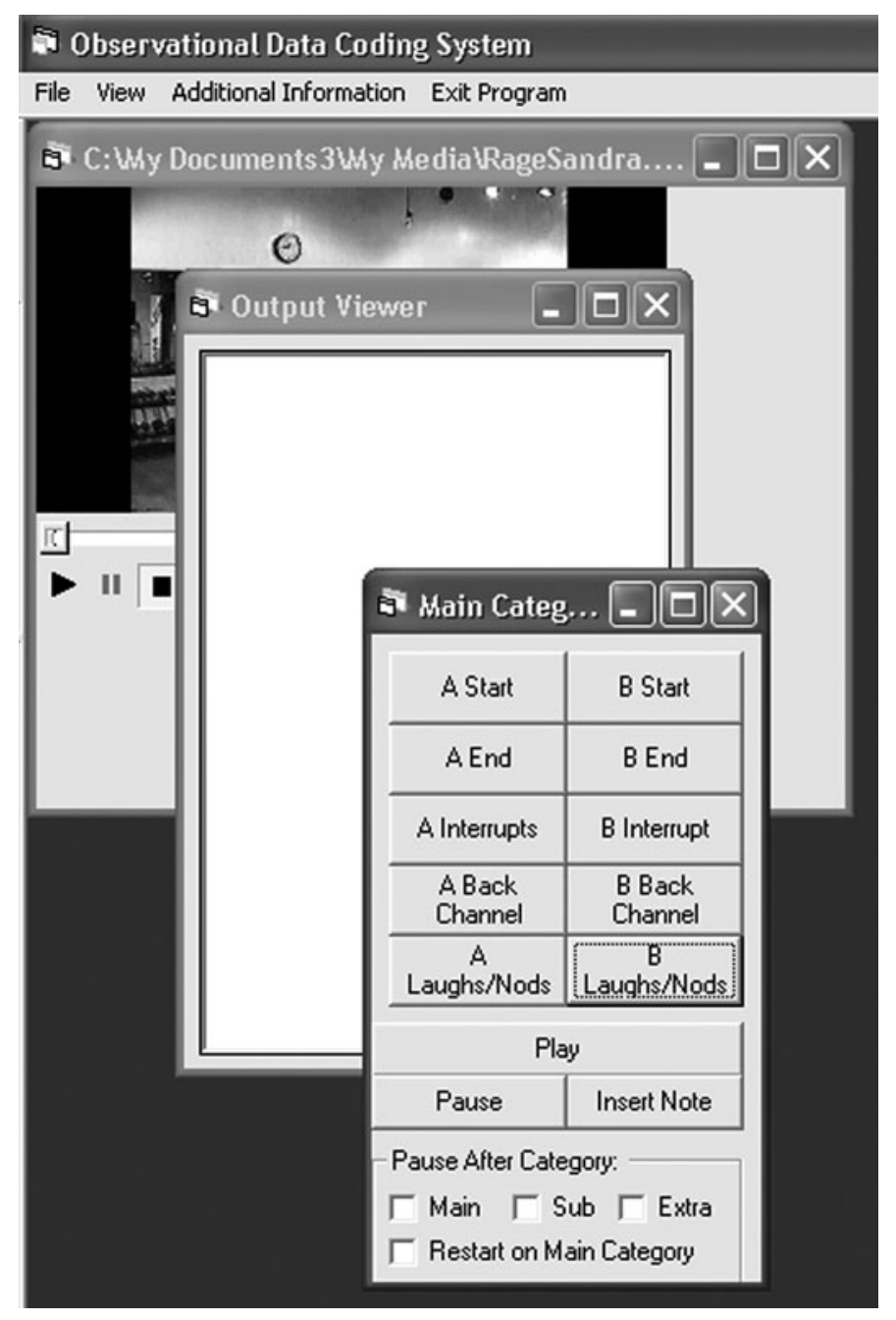

Figure 2. Main coding screen.

sized, for the ease and preference of the individual coder. Once situated, the coder, with the mouse, clicks on the play button on either the video viewer window or the main categories panel to begin the coding session.

Coders are able to view (or listen to) any portion of the file by dragging the player position slider to the left or right, to either rewind or fast forward the viewer. A pause button allows the coder to stop the media file whenever necessary. The program measures the media file in either frames or seconds. Therefore, a coder can go to a specific time or a specific frame by adjusting the slider. All of the coder's codes are time stamped, and the running clock on the player represents the length of time for the particular media file; it is not affected by the computer's own clock speed or by the duration of the coding session. Because the unexpected can occur, text notes for atypical events or questions for the researcher can be made during a coding session in the notes panel. The notes panel is activated by clicking on a button below the main categories panel. The note will be stamped with the current time or frame of the media file and written to the output file so that the researcher can know what the note is referring to in the media file and where it is. The note is then written to the text file.

\section{Data Retrieval, Transformation, and Analysis}

The data are written to a text file on the hard drive or other drive location (file name and location specified by the researcher in the control panel). The ODCS Webbased tutorial comes with instructions on how to transform this file into "real time," resulting in a chronological listing of the button clicks during the coding session. Instructions are included to transform the resulting file into one importable to Excel and SPSS. Reliability between coders can be determined by making a matrix chart (as has been discussed in Bakeman \& Gottman, 1997), using the tallies as they are provided in the program's output file. 


\section{An Example Using ODCS: Coding Conversation}

Although information about the specs of a program is important for any person interested in adopting a new piece of software, information about how a program actually has been used in a real study can be helpful not only to more completely introduce the potential of the software, but also to allow the user to envision his or her own use of it. To illustrate one particular use of this coding program, an overview of a study in which ODCS was used in conversational analysis will be presented.

Conversations, in terms of both their content and the process, have been of interest to psychologists, linguists, and communication specialists, among others. Of particular interest to the second author is the way in which people's cognitive schemata can affect a conversation with a partner who does or does not share the same schema (MacLin, 2000, 2005; MacLin \& Ginsburg, 2000). Studying conversation empirically, though, can be an onerous task. Videotaping conversations, transcribing them, and then coding and analyzing them are incredibly time consuming and labor intensive. Available software exists (an overview is provided below); however, most are complex and expensive. Out of necessity, ODCS was developed. Not only was it easy to use, but also it cut the time to do this project from several months to just several weeks. The resulting program served the need of this researcher well and, we felt, would be useful to others.

Study design and procedure. Female university students were recruited and asked to complete a variety of pretest measures, some of which assessed their schemas about college life. The measures were collected and scored by the researcher. From these respondents, two groups were selected to participate in the study: those whose scores indicated that they had a more academic orientation toward college life, and those whose scores indicated that they had a more social orientation toward college life. The participants were assigned to conversational dyads in which their schemas were mixed (social/acade$\mathrm{mic}$ ) or compatible (social/social or academic/acade$\mathrm{mic}$ ). The selected individuals were called to come to the laboratory for the experiment in pairs. They were then videotaped while engaging in a conversational task. The cover story was that they were there to help develop new freshman orientation materials. Their task was to discuss with their partner everything that they wished they had known about college before coming to college. They discussed this topic for $15 \mathrm{~min}$. It was hypothesized that those dyads in which the participants had compatible schemas would have different conversations in terms of content and style, as compared with those dyads with mixed schema partners.

Preparing the stimuli. The conversations were recorded on VHS tape. These were then transferred to a computer, using the Dazzle Digital Video Converter. This unit connects a standard VCR to a computer. Pushing "play" on the VCR (with the Dazzle software in ready mode) starts the encoding process. The resulting MPEG files were then burned to CD for storage pur- poses. ODCS, though, can access these files from the computer, CD, or DVD. Each 15-min dyadic interaction resulted in a 55-MB MPEG file. Thus, approximately 11 sessions fit on a single, 750-MB writable CD. Reasonably priced VHS/DVD recorders available on the market today make this entire encoding and storage process even easier.

Customizing ODCS. Detailed coding of particular aspects of the dyad's conversation, as well as the content of the conversation, was necessary. Main categories for this study were start and stop points for each speaker's talk, interruptions, nods, back-channel responding (i.e., utterances used to indicate that understanding has taken place-for example, "uh, huh" and "right"), and laughs. These event codes were found to be easily and accurately coded while the video segment was playing, and therefore, the option to have the video paused when making these codes was not selected in the control panel. Subcategory options appeared on the screen whenever the coder noted the end point of a given speaker's turn. These subcodes required more evaluative and thoughtful judgments regarding the speaker's turn as a whole, and therefore, when the coder was coding and rating these behaviors, the video segment was automatically paused, as previously determined in the control panel. These subcategories included whether or not the interruption (if any) in the previous utterance resulted in either a switch in turn or no switch in turn, shift or continuation of the previous speaker's train of talk, speaker affect, certain talk content, rating of talk content, and presence or absence of conversational repair (i.e., utterances that are used to correct understanding-e.g., "oh, no, what I meant was ...").

The coding program specified on the control panel thus made use of event codes (e.g., laughs or nods), durations (length of each speakers turn), Likert scales (animation rating), multiple choice options (affect and talk content), and yes/no options (shift and repair). Three passes of each 15-min interaction were required of the coder. The first pass was to actively view the session without coding, the second pass required the coder to code the behavior of the speaker on the left of the screen, and the third pass was to code the behavior of the speaker on the right of the screen. Screen prints showing the control panel and user interface for this study are available at the software Web site (http://www.uni.edu/ maclin/ software/odcs/).

Data coding, transformation, and analysis. Two independent coders blind to condition were trained to code the videotapes and make ratings of various aspects of the videotaped sessions. There was one main coder, who coded all the sessions, and a check coder, who coded $25 \%$ of the sessions (in order to evaluate reliability). The coders were given approximately $6 \mathrm{~h}$ of training. They first read several relevant articles and reviewed the detailed code book. The code book consisted of descriptions of the types of codes and examples of how various utterances would be coded (see the Appendix for an excerpt). They then met with the researcher individually to 
familiarize themselves with the installation and use of the coding program. Following this tutorial, the coder and the researcher coded one full session together, using the protocols in place (three passes and coding rules). They were then required to code a session by themselves. The data they collected from this session were then evaluated by the researcher, to ensure that all the coding rules and procedures were being followed correctly. One of the coders was given some additional training and then recoded the session.

Each 15-min session required approximately $90 \mathrm{~min}$ of coding time (taking into account starting and stopping the session, etc.). The codes were stored in the designated output file. Data from the two passes of coding sessions were sorted back into chronological order. The data were then imported into SPSS as tab-delimited files. From there, standard analyses could be conducted.

Study findings. To further explore the potential of this software, results from some analyses conducted using data outputted from ODCS are presented. Analyses from main category codes are presented first (and reflect the descriptive portion of the study).

Social participants. The participants categorized as social, had a mean turn length of $17.4 \mathrm{sec}$. They tended to provide the most verbal back-channel responding $(M=67.3)$ and took the most turns $(M=22.2$, as compared with academics, $M=17.6)$. They also had the smallest differences in mean turn length $(M=5.3 \mathrm{sec})$ and exhibited the lowest frequency of repair $(M=.007)$.

Academic participants. The participants categorized as academic in orientation toward college spoke the longest, with a mean turn length of $25.5 \mathrm{sec}$. They provided a low level of verbal back-channel responding $(M=52.6)$, a low level of nods $(M=26.1)$, and the fewest number of turns $(M=17.6)$. However, they spoke the longest (399.2 sec, or $6.7 \mathrm{~min}$ ).

Mixed-schema participants. Those participants who were in a mixed dyad (i.e., conversing with a partner who held a different schema about college life) had the shortest mean turn length $(M=19.1 \mathrm{sec})$ and the greatest amount of back-channel responding $(M=59.5)$. They also had the most number of turns $(M=21.7)$, as compared with compatible (same-schema) dyads $(M=17.8)$. Overall, they displayed the shortest difference in mean turn length from their partner $(M=5.9 \mathrm{sec})$.

Same-schema participants. The participants in the same-schema condition spoke less $(M=372.6 \mathrm{sec}$, or $6.21 \mathrm{~min})$, had longer turns $(M=23.5 \mathrm{sec})$, laughed more $(M=8.2)$, nodded the least $(M=26.8)$, back channeled the least $(M=56.0)$, and took the fewest turns $(M=17.8)$. Overall, they had the shortest conversations $(M=745.2 \mathrm{sec}, 12.4 \mathrm{~min})$ and exhibited the highest rate of repair $(M=.375)$.

Differences in conversation content were evaluated by analyzing codes derived out of the subcategory codes.

Schema mentions. Each turn was evaluated by the coder to assess whether any social or academic content had been discussed. Each turn was rated for the negative, positive, or neutral content of both academic and social information. A no-mention option was also available. Those participants with a social orientation were much more likely to communicate positively about social experiences in college when conversing with another socially oriented person, as compared with an academically oriented person ( $M=8.5$ vs. 2.5 , respectively). Academically oriented participants were much more likely to make no mention of academic information when conversing with socially oriented participants than when conversing with their fellow academically oriented participants ( $M=8.9$ vs. $M=3.6$, respectively).

ODCS provided a manageable, cost-effective way to code conversational data and prepare it for analysis. This is but one example of how this program can be used and is in no way limited to the coding of conversations. Researchers employing varying coding schemes, stimuli, and research questions have been consulted on the utility of ODCS for their research programs. The overwhelming consensus is that "if only" they had had ODCS available to them, they would have been able to use it in their area of study (e.g., mother-child interactions, negotiation behaviors, chat room discussions of bereaved parents, preschool teasing behaviors, rat behaviors in novel environments, etc.). In addition, ODCS has been successfully used in coding mock jury deliberations (H. Hosch, personal communication, 2000).

\section{Comparison With Other Software Packages}

Several coding and analysis programs are currently available; however, some researchers may find that ODCS has an advantage over the competition, due to the cost and availability advantages of this system. A brief overview of existing programs is provided for comparison purposes and to illustrate how ODCS might fit a variety of research needs.

There are several existing software packages available for the coding of observational data. The Observer VideoPro (Noldus, Trienes, Hendriksen, Jansen, \& Jansen, 2000; http://www.noldus.com) is probably the most comprehensive, providing collection, management, and analysis of observational data. However, the complexity and cost of this program may put it out of reach for some researchers.

Kahng and Iwata (1998) have provided a helpful review of 15 programs. Most of these programs are, however, designed for handheld or portable computers for use in field research, often for direct, "live" observation of humans or animals (e.g., Behavior Observer System, Behavioral Evaluation Strategy and Taxonomy, DATACAP, MOOSE [Tapp, Wehby, \& Ellis, 1995], and the Observer from Noldus Information Technology [Noldus, 1991], PROCODER [Tapp \& Walden, 1993]). ODCS is designed for evaluating previously collected observational sessions in the form of video, audio, or text files. PROCODER can also be used to code from videotape but requires time stamping the tape first. In addition, some programs require that buttons or keys be held down to record the durations of events (e.g., EventLog) or require keystrokes that do not intuitively relate to the code (e.g., MOOSES, the Observer). ODCS avoids these possibly confusing 
methods of coding by relying on a familiar, Windowsbased point-and-click system. Some programs have made use of the familiar Windows platform (e.g., Observer Video Pro and Qualrus's qualitative analysis program) but may still be cost prohibitive for many researchers.

In addition, some existing programs (e.g., the Observer) require data transformation processes (Bakeman \& Quera, 2000), because of the specialized nature of the coding program's data output files. The output of ODCS sessions is a text file and can be easily imported into other programs, unlike other packages that output data into specialized data files. Like most existing programs, ODCS data files require some transformations before they are exported to Excel or SPSS. There are several programs available to aid in the analysis of certain types of observational data; most notable are the sequential analysis programs of Bakeman and colleagues (Bakeman, 1983; Bakeman \& Gottman, 1997; Bakeman \& Quera, 1995; Dodd, Bakeman, Loeber, \& Wilson, 1981).

The most obvious differences between ODCS and most other existing behavioral coding packages are cost effectiveness and availability. The most inexpensive programs run from $\$ 700$ ( $\$ 200$ for students) to several thousand dollars, and some programs require installation and consultation by the software company prior to use. In contrast, ODCS is available for downloading free of charge on a Web site. There is little doubt that programs such as the Observer Video-Pro (Noldus et al., 2000) offer extensive observational and data analysis options, including customization services and individualized customer support. However, we feel that the design and cost effectiveness of ODCS will be useful to many a researcher whose project or pocketbook does not warrant so complex a system.

\section{Summary}

In short, this program provides a time-saving, flexible, and cost-effective way to code audio, video, image, or text media relevant to one's research. Code types, such as event codes, durations, Likert scales, multiple choice options, yes/no options, and many more, are possible with this program. Analyses of observations in the form of human or animal behaviors captured on video- or audiotape directly for the purposes of research or the systematic analysis of existing media (such as commercials, images, film clips, transcripts, or Internet material) are all possible. Researchers who are studying mock jury deliberations, mother-infant interactions, or children's play behaviors, evaluating the effectiveness of therapies, examining nonverbal communication or negotiation and bargaining strategies, or addressing many other topics, including animal behavior, might find this program useful.

We hope that this program not only will encourage researchers to use this time-saving method for their current work, but also will prompt researchers to bring out their shelved VHS data and reanalyze or ask different questions of their archived data.

\section{Availability}

The ODCS is currently available free for download from our Web site on the Internet (http://www.uni.edu/ $\sim$ maclin/software/odcs/). The download includes all of the Visual Basic libraries required to run the program on a non-VB-equipped computer, although we recommend that VB 6.0 be installed on the computer if possible, since the program may not be $100 \%$ compatible with the .NET framework. On the Web site, we provide, along with the program, documentation, installation procedures, and an on-line tutorial. Researchers using ODCS are requested to cite the present article whenever they report on their work.

\section{REFERENCES}

Allen, D. E., \& GuY, R. F. (1974). Conversation analysis: The sociology of talk. Paris: Mouton.

BAKEMAN, R. (1983). Computing lag sequential statistics: The ELAG program. Behavior Research Methods \& Instrumentation, 15, 530-535.

BaKeman, R., \& GotTMan, J. M. (1997). Observing interaction: An introduction to sequential analysis (2nd ed.). New York: Cambridge University Press.

BAKEMAN, R., \& Quera, V. (1995). Analyzing interaction: Sequential analysis with $S D I S \&$ \&SEQ. New York: Cambridge University Press.

BAKEMAN, R., \& QueRA, V. (2000). OTS: A program for converting Noldus Observer data files to SDIS files. Behavior Research Methods, Instruments, \& Computers, 32, 207-212.

DodD, P.W.O., BAKEMAN, R., LoEber, R., \& WiLSON, S.C. (1981). JOINT and SEQU: FORTRAN routines for the analysis of observational data. Behavior Research Methods \& Instrumentation, 13, 686-687.

Drew, P. (1995). Conversation analysis. In J. A. Smith, R. Harre, and L. Van Langenhove (Eds.), Rethinking methods in psychology (p. 505). London: Sage.

EDWARDS, J. A., \& LAMPERT, M. D. (1993). Talking data: Transcription and coding in discourse research. Hillsdale, NJ: Erlbaum.

FARRELL, A. D. (1991). Computers and behavioral assessment: Current applications, future possibilities and obstacles to routine use. Behavioral Assessment, 13, 159-179.

KaHNG, S. W., \& Iwata, B. A. (1998). Computerized systems for collecting real-time observational data. Journal of Applied Behavior Analysis, 31, 253-261.

LAMPERT, M. D., \& ERvin-TriPP, S. M. (1993). Structured coding for the study of language and social interaction. In J. A. Edwards \& M. D. Lampert (Eds.), Talking data: Transcription and coding in discourse research (pp. 169-206). Hillsdale, NJ: Erlbaum.

MacLin, M. K. (2000). Trading on each other's truths: The role of schemata in conversation (Doctoral dissertation, University of Nevada, Reno). Dissertation Abstracts International, 61(2-B), 1134 (AA19961131).

MACLIN, M. K. (2005). Schemas in conversation: A situated cognition approach. Manuscript submitted for publication.

MacLin, M. K., \& Ginsburg, G. P. (2000, February). Psst...Your schema is showing: How schemas emerge and operate in conversations. Poster presented at the First Annual Meeting of the Society for Personality and Social Psychology, Nashville, TN.

Noldus, L.P.J. J. (1991). The Observer: A software system for collection and analysis of observational data. Behavior Research Methods, Instruments, \& Computers, 23, 415-429.

Noldus, L.P. J.J., Trienes, R. J. H., Hendriksen, A. H. M., Jansen, H., \& JANSEN, R. G. (2000). The Observer Video-Pro: New software for the collection, management, and presentation of time-structured data from videotapes and digital media files. Behavior Research Methods, Instruments, \& Computers, 32, 197-206.

Potter, J., \& Wetherell, M. (1995). Discourse analysis. In J. A. Smith, R. Harré, \& L. van Langenhove (Eds.), Rethinking methods in psychology (pp. 80-92). London: Sage. 
TAPP, J., \& WALDEN, T. (1993). PROCODER: A professional tape control, coding, and analysis system for behavioral research using videotape. Behavior Research Methods, Instruments, \& Computers, 25, 53-56.

TAPP, J., WehBy, J., \& Ellis, D. (1995). A multiple option observation system for experimental studies: MOOSES. Behavior Research Methods, Instruments, \& Computers, 27, 25-31.

\section{NOTES}

1. http://www.microsoft.com/windowsxp/using/moviemaker/21/ intro.mspx.

2. http://www.microsoft.com/windowsxp/using/moviemaker/default. mspx.

\section{APPENDIX \\ Codebook Excerpt}

An interruption occurs when one speaker attempts to take the floor before the other speaker has finished his or her utterance. It does not need to be a full sentence. Back channels (e.g., uh, huh) do not count as interruptions, even when they are a few words long (e.g., "I know what you mean"; there is no intent to take the floor). If Speaker A is talking and Speaker B attempts to take the floor and does not succeed, it would look like this (information in parentheses indicates the coding button that would be used in the program):

Speaker A: (“A starts”) I'm having a hard time dealing with my family and studying, it's a real bear, I still feel like I need to make home cooked meals, and I feel-

Speaker B: ("B interrupts") yah, but you shouldn't fe-

Speaker A: feel pulled in a million directions. ("A ends")

Speaker B: ("B starts") yah, but you shouldn't feel that way, school is an important priority. ("B ends")

This sequence indicates that there was an interruption with no switch in turn. Here is the same interaction, but where the interruption leads to a switch in turn:

Speaker A: (“A starts”) I'm having a hard time dealing with my family and studying, it's a real bear, I still feel like I need to make home cooked meals, and I feel-("A ends")

Speaker B: ("B interrupts") ("B starts") yah, but you shouldn't feel that way, school is an important priority. ("B ends")

Speaker A: ("A starts") yah, but I feel pulled in a million directions. ("A ends")

Because the speakers are coded separately, when the data are resorted back into A-B exchanges, this pattern will be apparent, indicating whether it was an interruption that resulted in a switch in turn or not.

(Manuscript received November 16, 2004;

revision accepted for publication May 10, 2005.) 